\section{AB1211 A RETROSPECTIVE ANALYSIS OF LABORATORY ABNORMALITIES FOUND DURING METHOTREXATE MONITORING AND THE COST IMPLICATIONS}

Sadriye Eriksson, Arslan Sidhu, Tom Walton. Colchester Hospital, East Suffolk and North Essex NHS Foundation Trust, Rheumatology, Colchester, United Kingdom

Background: Methotrexate (MTX) remains the first line treatment in majority of cases of inflammatory arthritis. The current British Society of Rheumatology (BSR) Guidelines suggest checking Full blood Count (FBC), ALT/AST, Albumin, Creatinine at $0,2,4,6,10,14,18$ weeks and then 12 weekly. Despite this close monitoring recommendation, MTX is generally considered a safe drug by Rheumatologists and its use has grown significantly over last two decades.

Objectives: To evaluate the incidence of liver, renal and haematological toxicities during Methotrexate treatment and calculate the cost implications.

Methods: 101 patients (30 males 71 females) (Age 40-89 year Mean 66.5 year) prescribed MTX were randomly selected and retrospective analysis was performed. 91 Rheumatoid arthritis, 8 Psoriatic arthritis and 2 patients had Undifferentiated Inflammatory Arthritis. 20 patients had Early Inflammatory Arthritis ( $<1$ year) and 81 had established disease. 24 patients were on MTX for $<1$ year. Average dose of MTX in our cohort was $15 \mathrm{mg}$ once weekly (Min $7.5 \mathrm{mg}$ Max $25 \mathrm{mg}$ ). All patients were on folate supplementation. Blood investigations over last 1 year were reviewed individually for all patients. Severe Liver toxicity (LS) was defined as ALT/AST >100 U/l; unexplained reduction in albumin $<30 \mathrm{~g} / \mathrm{l}$ and mild liver toxicity (LM) was between normal and LS values. Severe haematological toxicity (HS) was defined as WCC $<3.5 \times 10^{9} / \mathrm{l}$, MCV $>105 \mathrm{fL}$, Neut $<1.6 \times 10^{9} / \mathrm{l}, \mathrm{PLT}<140 \times 10^{9} / \mathrm{l}$ unexplained eosinophilia $>0.5 \times 10^{9} / \mathrm{l}$ and mild haematological toxicity (HM) was between normal and HS. Severe renal toxicity (RS) was defined as Creatinine increase $>30 \%$ over 12 months and/or calculated GFR $<60 \mathrm{ml} / \mathrm{min}$. Mild renal toxicity (RM) was between normal and RS.

Results: Over 1 year, 101 patients had total of 609 blood tests for MTX monitoring (Min 1 Max 17). FBC, Liver Function Test, Urea \& Electrolytes, CRP and ESR each cost £8.54. LS was found in 4 patients, LM in 15, HM in 3 and RM in 2 patients. MTX was stopped in all 4 patients with LS. Mild toxicities (LM, HM and RM) recovered after close monitoring or reduction in dose. 5 patients with mild toxicities were on MTX for $<1$ year. Rest of the patients were on stable dose of MTX for $>1$ year. All patients with severe and mild toxicities did not have any significant comorbidities compared to rest of the patients. It was calculated that detecting one LS cost $(609 \times 8.54) / 4=£ 1300.2$. Similarly one LM cost £346.7, one HM £1733.6 and one RM £2600.43. These costs does not include travel expenses, parking charges and time off work for appointments in phlebotomy.

Conclusion: Our cohort shows that mild liver toxicity is the most commonly found abnormality during MTX monitoring and patients on stable doses still need monitoring for liver toxicity. Haematological and renal toxicities are much less common and a more relaxed monitoring schedule may be acceptable for these parameters. Regular monitoring for inflammatory markers (CRP, ESR) causes an extra cost burden. The direct cost for identifying each abnormality is considerable.

\section{REFERENCES}

[1] Ledingham J, Gullick N, Irving K, Gorodkin R, Aris M, Burke J, et al. BSR and BHPR guideline for the prescription and monitoring of non-biologic disease-modifying anti-rheumatic drugs. Rheumatology. 2017 Feb 27;56 (6):865-868. kew479.

Disclosure of Interests: None declared

DOI: 10.1136/annrheumdis-2019-eular.3790

\section{AB1212 POLYPHARMACY AND OUTPATIENT HEALTHCARE USE IN RHEUMATOID ARTHRITIS: PATTERNS AND ASSOCIATIONS}

George E. Fragoulis ${ }^{1}$, Savvas Psarelis ${ }^{2}$, Christina Flourou ${ }^{3}$, Andreas Tofarides ${ }^{3}$, Elena Nikiphorou ${ }^{4}{ }^{1}$ University of Glasgow, Institute of infection, immunity and inflammation, Glasgow, United Kingdom; ${ }^{2}$ Nicosia General Hospital,

Rheumatology, Nicosia, Cyprus; ${ }^{3}$ Nicosia General Hospital, Internal Medicine Department, Nicosia, Cyprus; ${ }^{4}$ King's College, Department of Inflammation Biology, London, United Kingdom

Background: Polypharmacy is a considerable problem in people with rheumatic diseases, including rheumatoid arthritis (RA), related amongst others to worse disease outcomes and increased cost for the health-system (1).

Objectives: To assess polypharmacy in RA and usage of the health-care system (outpatient clinics) in a real-world setting.

Methods: Medical records of 170 consecutive RA patients from a large outpatien service of a central hospital were retrospectively reviewed. Demographic characteristics, treatment for RA and comorbidities as well as frequency and type of visits to any outpatient services were recorded. The latter included rheumatologists, "medical specialties" doctors (general physicians, cardiologists, respiratory physicians, oncologists, dermatologists, gastroenterologists, hematologists, nephrologists and neurologists) and "surgical specialties" (general surgery, orthopedics, neurosurgery, urology, ophthalmology, ENT, maxillofacial and vascular surgery). Disease duration was defined as the time between RA diagnosis and the end of the study (May 2018). Univariable and multivariable analyses were performed (Table).

Results: Data from 170 RA patients $(77.1 \%$ female) with a mean \pm SD age of: $62.1 \pm 13.7$ years and disease duration: $87.8 \pm 22.0$ months were recorded. The median number of non-rheumatic drugs receieved througouht disease duration was 3 . Only $7 \%$ of the patients were not receiving any additional drugs, while $15.3 \%, 40.6 \%$ and $56.4 \%$ had received 1,2 and 3 non-rheumatic drugs, respectively. The most commonly used non-rheumatic drugs were, anti-hypertensive, anti-osteoporotic and lipid-lowering drugs. Higher total number of drugs correlated with age of the patient and longer disease duration. Methotrexate (MTX) experienced or biologic-naïve patinets, had received a larger number of nonrheumatic drugs compared to those who had not received methotrexate or were biologic-experienced. Multivariable analysis confirmed age and exposure to methotrexate/biologics to be positively and negatively association respectively, with the number of non-rheumatic drugs received (Table)

Number of visits to rheumatologists/year were: median (range) 2.9 (0.5 13.6). The number of visits was correlated with age of the patients, disease duration and number of non-rheumatic drugs received. Multivariable analysis, identified, disease duration, number of non-rheumatic drugs received and being MTX-naive as predictors of number of rheumatology visits (Table).

Number of visits to non-rheumatologists/year were: median (range) 1.8 (0.0 - 13.7). Visits to "internal medicine" doctors were more common than in "surgical specialties" doctors $(p<0.0001)$. Number of visits to nonrheumatologists was correlated with age, disease duration, and with the number of non-rheumatic drugs received. Multivariable analysis confirmed only the association with the total number of drugs but revealed also that being MTX-naive was also associated with more frequent visits to non-rheumatologists.

Conclusion: The majority of RA patients received more than 3 (non-RA related) drugs. This increased with older age and associated with more frequent visits to rheumatology and other specialties.

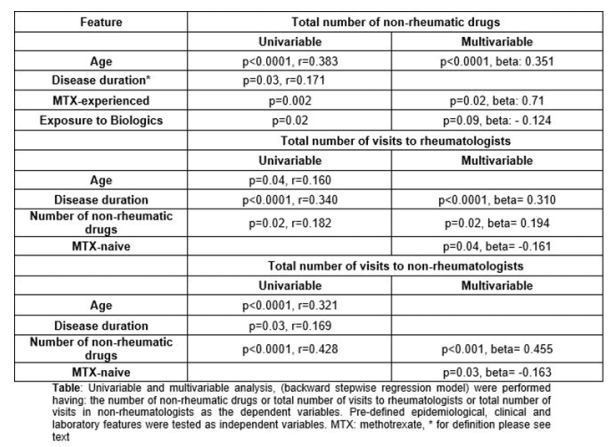

Table 1

\section{REFERENCES}

[1] Filkova M, et al. J Rheumatol. 2017 Dec;44:1786-1793

Disclosure of Interests: None declared

DOI: 10.1136/annrheumdis-2019-eular.774 